\title{
A DUPLICAÇÃO DO NÚMERO DE MÃOS DE SINAIS DA LIBRAS E SEUS EFEITOS SEMÂNTICOS
}

\author{
LA DUPLICACIÓN DEL NÚMERO DE MANOS DE SEÑALES DE LIBRAS Y SUS EFECTOS \\ SEMÁNTICOS
}

\section{THE DUPLICATION OF THE NUMBER OF HANDS IN SIGN LANGUAGE, AND ITS SEMANTIC EFFECTS}

\author{
André Nogueira Xavier* \\ Plínio Almeida Barbosa** \\ Universidade Estadual de Campinas
}

\begin{abstract}
RESUMO: Segundo Xavier (2006), há sinais na língua brasileira de sinais, libras, que são tipicamente produzidos com uma mão e outros com duas. Entretanto, trabalhos recentes documentam a produção, com duas mãos, de sinais normalmente feitos com uma, e vice-versa (XAVIER, 2011; XAVIER; BARBOSA, 2013). Este trabalho objetiva discutir 27 sinais da libras, tipicamente realizados com uma mão, que, ao serem articulados com duas, apresentam mudança em seu significado. Os dados discutidos aqui, apesar de originalmente coletados a partir de observações de sinalizações espontâneas de diferentes usuários da libras, foram eliciados de duas sinalizantes surdas em sessões separadas. Depois de apresentar as duas formas (com uma e duas mãos) dos sinais selecionados, as sinalizantes foram solicitadas a produzir exemplos de uso para cada uma delas. Os resultados mostraram que a duplicação no número de mãos, em alguns casos para um mesmo sinal, pode se dar por diferentes fatores, entre eles, pluralidade, aspecto e intensidade.
\end{abstract}

PALAVRAS-CHAVE: libras; número de mãos; duplicação; efeitos semânticos.

RESUMEN: Según Xavier (2006), existen señales en la Lengua de Señas Brasilera (Libras) que típicamente se producen con una mano y otras con dos. No obstante, trabajos recientes documentan la producción con dos manos de señales que normalmente se realizan con una y viceversa (Xavier, 2011; Xavier; Barbosa, 2011, 2013). Este trabajo pretende analizar 27 señales de Libras que típicamente se articulan con una mano y que, cuando son producidas con dos, muestran un cambio en su significado. Los datos discutidos aquí, originalmente colectados a partir de observaciones de señales espontáneas de diferentes usuarios de Libras, fueron obtenidos de dos usuarias sordas en sesiones distintas. Tras mostrar las formas unimanuales y bimanuales de las señas seleccionadas, el experimentador solicitó a las usuarias sordas que diesen ejemplos de uso para cada una de ellas. Los resultados muestran que la duplicación del número de manos puede ser causada por distintos factores (como pluralidad, aspecto y intensidad), incluso para una misma señal.

PALABRAS CLAVE: Libras, número de manos, duplicación, efectos semánticos.

ABSTRACT: According to Xavier (2006), there are signs in the Brazilian sign language (Libras) that are typically developed with one hand, while others are made by both hands. However, recent studies document the communication, with both hands, of signs which usually use only one hand, and vice-versa (XAVIER, 2011; XAVIER, 2013; BARBOSA, 2013). This study aims the discussion of 27 Libras' signs which are typically made with one hand and that, when articulated with both hands, present changes in their meanings. The data discussed hereby, even though originally collected from observations of spontaneous signs from different Libras' users, have been elicited by two deaf patients in distinct sessions. After presenting the two forms of the selected signs (made with one and two hands), the patients were asked to create examples of use for each of the signs. The results proved that the duplication of hands, at least for the same signal in some cases, may happen due to different factors (such as plurality, aspect and intensity).

KEYWORDS: Libras; hand number; duplication; semantic effects.

\section{INTRODUÇÃO}

As línguas de sinais, usadas pelas diversas comunidades surdas espalhadas pelo mundo, se diferenciam marcadamente das línguas orais pela sua modalidade de percepção e de produção. Especificamente, os itens

\footnotetext{
* Doutor em Linguística na Universidade Estadual de Campinas (Unicamp). Email: andre.xavier.unicamp@gmail.com.

** Doutor em Signal-Image-Parole/Option Parole pelo Institut de la Communication Parlée e Institut National Polytechnique de Grenoble, França. Professor Associado do Departamento de Linguística do Instituto de Estudos da Linguagem (Unicamp) e pesquisador do Conselho Nacional de Desenvolvimento Científico (CNPq) (301387/2011-7). Email: pabarbosa.unicampbr@gmail.com.
} 
lexicais dessas línguas, tradicionalmente chamados de sinais, são percebidos pela visão e majoritariamente articulados pelas mãos ${ }^{1}$. As mãos são geralmente descritas na literatura sobre as línguas de sinais por meio dos termos ativo ou passivo e dominante ou não-dominante (BATTISON, 1978). Designa-se como ativa a mão que apresenta movimento e passiva a que fica estacionada, servindo de ponto de articulação para a ativa.

Descreve-se como dominante a mão preferida para realizar sinais de uma mão e desempenhar o papel de mão ativa em certos sinais feitos com duas mãos, e não-dominante a preterida para as mesmas funções.

Em virtude de as línguas de sinais apresentarem sinais tipicamente articulados com uma mão e outros com duas, Klima e Bellugi (1979) propuseram a inclusão do parâmetro arranjo de mãos no rol das unidades sublexicais ${ }^{2}$ empregadas na análise fonológica dos sinais da língua de sinais americana (ASL, do inglês American Sign Language). Os autores também fundamentaram a inclusão desse parâmetro na existência de pares mínimos na ASL em que o contraste semântico entre os sinais do par se estabelece unicamente com base no número de mãos ${ }^{3}$. Além de descrever se os sinais são produzidos com uma ou duas mãos, o parâmetro arranjo de mãos também tem como função especificar, no caso de sinais realizados com duas mãos, se ambas são ativas ou se uma delas é ativa e a outra passiva.

A literatura, majoritariamente sobre a ASL, documenta alguns processos relacionados à dinâmica das mãos na produção de sinais. Entre esses processos está: a troca de dominância (dominance shift), a antecipação e a perseveração da mão não-dominante de sinais feitos com duas mãos em sinalização corrente, o espelhamento (mirrowing) da mão domimante pela mão não-dominante durante a produção de sinais de uma mão e a simultaneidade (ou co-produção) de dois sinais.

A troca de dominância consiste no emprego da mão não-dominante em situações em que a mão dominante normalmente é usada - na realização de sinais de uma mão e no desempenho do papel de mão ativa em sinais feitos com duas mãos (BATTISON, 1974)4. A antecipação e a perseveração, por sua vez, consistem, respectivamente, em antecipar ou perseverar a mão não-dominante envolvida na produção de sinais feitos com duas mãos durante a realização de um ou mais sinais tipicamente articulados apenas com uma (LIDDELL; JOHNSON, 1989; SANDLER, 1993). Já o espelhamento consiste na cópia das características e atividades articulatórias da mão dominante pela mão não-dominante quando esta não está participando da realização de um sinal e, portanto, encontra-se em repouso no colo ou na altura do peito (NILSSON, 2007). Por fim, a coprodução se refere à articulação de dois sinais ao mesmo tempo: um em cada mão. Esses casos se caracterizam por apresentar a mão não-dominante realizando, estaticamente, uma parte de um sinal anterior ou seguinte enquanto a dominante articula outro sinal (LIDDELL, 2003; HENDRICKS, 2007; VERMEERBERGEN; DEMEY, 2007).

Além desses, Johnston e Schembri (1999) documentam que na língua de sinais australiana (Auslan) sinais tipicamente articulados com uma mão podem, às vezes, ser produzidos com duas e sinais normalmente feitos com duas mãos podem ser, por vezes, produzidos com uma. Os autores designam o primeiro processo como duplicação (doubling) e o segundo como unificação (singling).

Com base nos dados documentados por Xavier (2011) e Xavier e Barbosa (2013), ambos os processos ocorrem na libras e podem ou não alterar o significado do sinal. Entre os sinais em que a mudança no número de mãos tem efeitos semânticos, os autores só identificaram casos de duplicação. Porém, entre os sinais em que não há mudança de significado, eles identificaram tanto casos de duplicação quanto de unificação (Tabela 1).

\footnotetext{
${ }^{1}$ A preferência pelas mãos para a articulação de sinais é evidenciada na libras pelo estudo de Xavier (2006). Dentre os 2.274 sinais analisados, o autor identificou apenas cinco unicamente produzidos não-manualmente, especificamente por meio de movimentos da boca, da língua ou de uma das bochecha.

${ }^{2}$ Os parâmetros propostos até então abrangiam a configuração de mão, a orientação da palma, a localização e o movimento.

${ }^{3}$ Para Klima e Bellugi (1979), o arranjo das mãos é um subparêmetro do parâmetro configuração de mão. Eles o tratam assim, por considerarem que, em comparação aos parâmetros principais, entre os quais está a configuração de mão, ele distingue um número bem menor de sinais semanticamente não relacionados.

${ }^{4}$ Esse fenômeno só é possível porque, segundo Battsion (1978), a escolha da mão, direita ou esquerda, não é distintiva de significado.
} 
Tabela 1: Sumário dos casos de duplicação e unificação com e sem efeito semântico com base nos dados de Xavier (2011) e Xavier e Barbosa (2013)

\begin{tabular}{c|c|c} 
& Com efeito semântico & Sem efeito semântico \\
\hline Duplicação & $\boldsymbol{x}$ & $\mathbf{x}$ \\
\hline Unificação & & $\boldsymbol{*}$
\end{tabular}

Xavier e Barbosa (2013) citam entre os fatores semânticos que podem motivar a duplicação a expressão de pluralidade, aspecto ou intensidade. Os autores também apontam que é possível que um mesmo sinal sofra alteração em seu número de mãos em função de diferentes fatores semânticos, a depender do contexto de uso. Segundo eles, um determinado sinal, tipicamente feito com uma mão, pode ser realizado com duas para expressar, por exemplo, pluralidade em um caso e intensidade em outro.

No estudo descrito a seguir, foram eliciados de duas sinalizantes surdas exemplos de uso para os casos levantados por Xavier (2011) e Xavier e Barbosa (2013) em que a duplicação do número de mãos tem diferentes efeitos semânticos. Nosso objetivo foi verificar, através da análise do significado das formas produzidas com duas mãos em contexto, se, de fato, mais de uma motivação semântico pode ter motivado o processo em questão.

\section{MÉTODO}

\subsection{Sujeitos}

Participaram do experimento duas sinalizantes surdas bilíngues (libras e português). Uma, doravante SL, tinha 44 anos quando participou do experimento e a outra, doravante RA, 42. Ambas nasceram em famílias ouvintes e, consequentemente, aprenderam libras tardiamente: a primeira aos seis e a segunda aos sete anos de idade. As duas passaram a infância e a juventude na cidade de São Paulo. Atualmente, apenas uma reside nessa cidade. A outra mora, há aproximadamente 6 anos, em Indaiatuba, interior do estado de São Paulo. As duas sinalizantes têm formação superior e atuam como professoras de libras para ouvintes.

\subsection{Estímulos}

Os 23 sinais usados como estímulos neste estudo provêm do trabalho de Xavier e Barbosa (2013), onde eles são categorizados com base no fator semântico que, segundo os autores, motiva a duplicação do seu número de mãos. Os estímulos, listados na Tabela 2 , são aqui designados pela glosa da forma mais comum, em geral, a de uma mão.

Tabela 2: Sinais empregados como estímulos em sua forma com uma e duas mãos ativas

\begin{tabular}{c|c} 
Estímulos & Total \\
\hline ACUSAR, APRENDER, ÁRVORE, & 23 \\
AVISAR, BEIJAR, CAFÉ, CHAMAR, & \\
COLAR, COMER, DINHEIRO, & \\
ENTENDER, FALAR, IGNORAR, & \\
IMAGINAR, INVENTAR, IR-EMBORA, & \\
MAGR@, DIFERENTE, OPINIÃO, O- & \\
QUE, PAGAR, RIR 5 , VER. &
\end{tabular}

${ }^{5}$ Incluímos no estudo tanto a forma de RIR em que ambas as mãos se movem simultaneamente para os lados ou para baixo no espaço em frente ao corpo do sinalizante, quanto uma outra em que elas ficam lado a lado, com as palmas voltadas para dentro e em frente ao queixo. 


\subsection{Procedimentos}

Solicitamos às duas participantes surdas exemplos de uso para os 23 sinais listados na Tabela 2. Esses exemplos foram produzidos logo depois que o primeiro autor sinalizou para elas a forma feita com uma e duas mãos de cada um dos sinais ${ }^{6}$ listados na Tabela 2.

A participação das sinalizantes surdas se deu em sessões separadas com duração média de 12 minutos. As sessões foram registradas em vídeo por meio de uma câmera Samsung modelo Hyper DIS 65x intelli-zoom. A análise qualitativa dos dados foi realizada com o software livre ELAN (Eudico Language Annotator) ${ }^{7}$, que permitiu segmentar os vídeos nas porções correspondentes a cada um dos 23 sinais.

\section{RESULTADOS}

A análise dos dados obtidos mostrou que os dois sujeitos coincidiram no significado com que empregaram 18 das 23 formas de duas mãos dos sinais selecionados para o estudo. Isso sugere que para ambos a duplicação do número de mãos majoritariamente se deu pelos mesmos fatores.

Entre esses fatores está a pluralidade que, como mostra a Tabela 3, pode incidir sobre a própria entidade designada pelo sinal ou sobre um de seus argumentos, no caso de ele ser um predicador. Em termos de algumas de suas características formais, nota-se que o movimento das mãos na forma duplicada pode ser simultâneo ou alternado e ainda reto, sem trajetória ${ }^{8}$ ou circular.

Tabela 3: Sinais produzidos com duas mãos para a expressão de pluralidade

\begin{tabular}{|c|c|c|c|c|c|c|c|}
\hline \multirow[t]{2}{*}{ Sinal } & \multicolumn{3}{|c|}{ Pluralidade do referente } & \multicolumn{4}{|c|}{ Movimento } \\
\hline & $\begin{array}{l}\text { Entidade } \\
\text { designada }\end{array}$ & Sujeito & Complemento & Simultâneo & Alternado & Reto & Circular \\
\hline ÁRVORE & $\boldsymbol{x}$ & & & $\boldsymbol{x}$ & $\boldsymbol{*}$ & $\mathbf{x}$ & \\
\hline APRENDER & & & $x$ & & $\boldsymbol{x}$ & & $x$ \\
\hline COLAR & & $\boldsymbol{x}$ & & $\boldsymbol{x}$ & $\boldsymbol{x}$ & & $x$ \\
\hline $\begin{array}{c}\text { IR- } \\
\text { EMBORA }\end{array}$ & & $\boldsymbol{x}$ & & & $\boldsymbol{x}$ & $x$ & \\
\hline INVENTAR & & & $\mathbf{x}$ & & $\boldsymbol{*}$ & $\mathbf{x}$ & \\
\hline OPINIÃO & $\boldsymbol{*}$ & & & & $\boldsymbol{x}$ & & $x$ \\
\hline PAGAR & & & $x$ & & $\boldsymbol{x}$ & & $x$ \\
\hline
\end{tabular}

Um subconjunto dos sinais que têm seu número de mãos duplicado para expressar pluralidade se constitui dos chamados verbos direcionais ou indicadores. Esses verbos são assim chamados porque têm sua forma modificada em virtude das diferentes localizações no espaço de sinalização a que seus referentes podem estar associados. A Tabela 4 mostra que, nesses verbos, a duplicação só acontece quando seu sujeito ou complemento se referem à segunda ou terceira pessoa do plural. Em relação às suas características formais, observa-se que a versão do sinal com o número de mãos duplicado sempre apresenta movimento simultâneo das mãos quando expressa pluralidade de complemento ${ }^{9}$, e movimento simultâneo e/ou alternado, quando expressa a pluralidade do

\footnotetext{
${ }^{6}$ Para um estudo com método semelhante, ver Börstell (2011).

${ }^{7}$ Disponível em <http://tla.mpi.nl/tools/tla-tools/elan/>.

${ }^{8}$ Movimentos sem trajetória são aqueles em que a mão se move não por se deslocar no espaço, mas por mudar de uma configuração ou orientação para outra (LIDDELL; JOHNSON, 1989).

${ }^{9}$ Os dois sujeitos estranharam a forma de BEIJAR e IGNORAR com o número de mãos duplicado para expressar pluralidade de seus sujeitos. Por essa razão, na Tabela 3, essas formas são marcadas com um ponto de interrogação. Cabe dizer que dessas duas apenas a de IGNORAR foi efetivamente vista em uma sinalização espontânea.
} 
sujeito.

Tabela 4: Verbos indicadores que sofreram duplicação do número de mãos para a expressão de pluralidade

\begin{tabular}{|c|c|c|c|c|c|c|c|c|}
\hline \multirow[b]{2}{*}{ Sinal } & \multicolumn{2}{|c|}{$\begin{array}{c}1^{\mathrm{a}} \text { pessoa do singular } \rightarrow 2^{\mathrm{a}} \\
\text { ou } 3^{\mathrm{a}} \text { pessoa do plural }\end{array}$} & \multicolumn{2}{|c|}{ Movimento } & \multicolumn{2}{|c|}{$\begin{array}{c}2^{\mathrm{a}} \text { ou } 3^{\mathrm{a}} \text { pessoa do plural } \\
\rightarrow 1^{\mathrm{a}} \text { pessoa do singular }\end{array}$} & \multicolumn{2}{|c|}{ Movimento } \\
\hline & Plu. Suj. & Plu. Comp. & Simul. & Altern. & Plu. Suj. & Plu. Comp. & Simul. & Altern. \\
\hline ACUSAR & & $\boldsymbol{x}$ & $\mathbf{x}$ & & $x$ & & $\boldsymbol{x}$ & \\
\hline AVISAR & & $x$ & $\boldsymbol{x}$ & & $\boldsymbol{x}$ & & $\boldsymbol{x}$ & $\boldsymbol{x}$ \\
\hline BEIJAR & & $x$ & $\boldsymbol{x}$ & & $?$ & & $\boldsymbol{x}$ & \\
\hline CHAMAR & & $x$ & & $\boldsymbol{x}$ & $\boldsymbol{x}$ & & & $\boldsymbol{x}$ \\
\hline IGNORAR & & $x$ & $\boldsymbol{x}$ & & ? & & $x$ & \\
\hline
\end{tabular}

Em um outro conjunto de dados, manifestou-se como fator para a duplicação do número de mãos a expressão de intensidade. No que diz respeito às suas características formais, as versões intensificadas, assim como as duplicadas por pluralidade, apresentam também diferentes tipos de movimento: simultâneo ou alternado e reto ou circular (Tabela 5).

Tabela 5: Sinais que sofreram duplicação do número de mãos para a expressão de intensidade

\begin{tabular}{|c|c|c|c|c|}
\hline \multirow[t]{2}{*}{ Sinal } & \multicolumn{4}{|c|}{ Movimento } \\
\hline & Simultâneo & Alternado & Reto & Circular \\
\hline COMER & & $x$ & & $\boldsymbol{x}$ \\
\hline DINHEIRO & $\boldsymbol{x}$ & & $\boldsymbol{x}$ & \\
\hline FALAR & & $\boldsymbol{x}$ & & $x$ \\
\hline IMAGINAR & $\boldsymbol{x}$ & & $\boldsymbol{x}$ & \\
\hline O-OUE & & $\boldsymbol{x}$ & & $\boldsymbol{*}$ \\
\hline $\mathrm{RIR}^{10}$ & $x$ & & $\boldsymbol{x}$ & \\
\hline
\end{tabular}

Embora a duplicação do número de mãos possa ser explicada pela expressão de pluralidade ou intensidade em $78 \%$ dos dados, em três sinais (13\%), a saber, CAFÉ, ENTENDER e VER, esse processo parece ter sido motivado por fatores diferentes (Tabela 6).

Tabela 6: Sinais que sofreram duplicação do número de mãos por diferentes fatores

\begin{tabular}{c|c|c|c|c}
\multirow{2}{*}{ Sinal } & \multicolumn{2}{|c|}{ Duplicação } & \multicolumn{2}{c}{ Movimento } \\
\cline { 2 - 5 } & Fator 1 & Fator $\mathbf{2}$ & Simultâneo & Alternado \\
\hline CAFÉ & Pluralidade do sujeito & $\begin{array}{c}\text { Aspecto iterativo e/ou } \\
\text { intensidade }\end{array}$ & & $\mathbf{*}$ \\
\hline ENTENDER & Completude da ação & Pluralidade do sujeito & $\mathbf{*}$ & $\mathbf{*}$ \\
\hline VER & $\begin{array}{c}\text { Tamanho do objeto e/ou não } \\
\text { pontualidade do olhar }\end{array}$ & $\begin{array}{c}\text { Pluralidade do sujeito } \\
\mathbf{*}\end{array}$ & & $\mathbf{*}$
\end{tabular}

Como mostra a Tabela 6, nos dados obtidos para este estudo, o sinal CAFÉ teve seu número de mãos duplicado por dois fatores: a expressão de pluralidade de seu sujeito e a iteração e/ou intensidade da ação de tomar café. $O$ primeiro caso foi observado no exemplo de uso criado por SL. Ela empregou a versão com uma mão de CAFÉ quando simulou convidar uma única pessoa para tomar café e a versão com duas quando simulou estender o mesmo convite a várias pessoas (Figura 1a). O segundo caso, por sua vez, ocorreu no exemplo criado por RA, no qual a forma de duas mãos de CAFÉ foi empregada quando esta se referiu a uma pessoa que toma café, um

${ }^{10}$ Aqui se está considerando a forma feita com duas mãos articulada no espaço em frente ao corpo do sinalizante. 
atrás do outro, ao longo de todo o dia, portanto, excessivamente (Figura 1b).
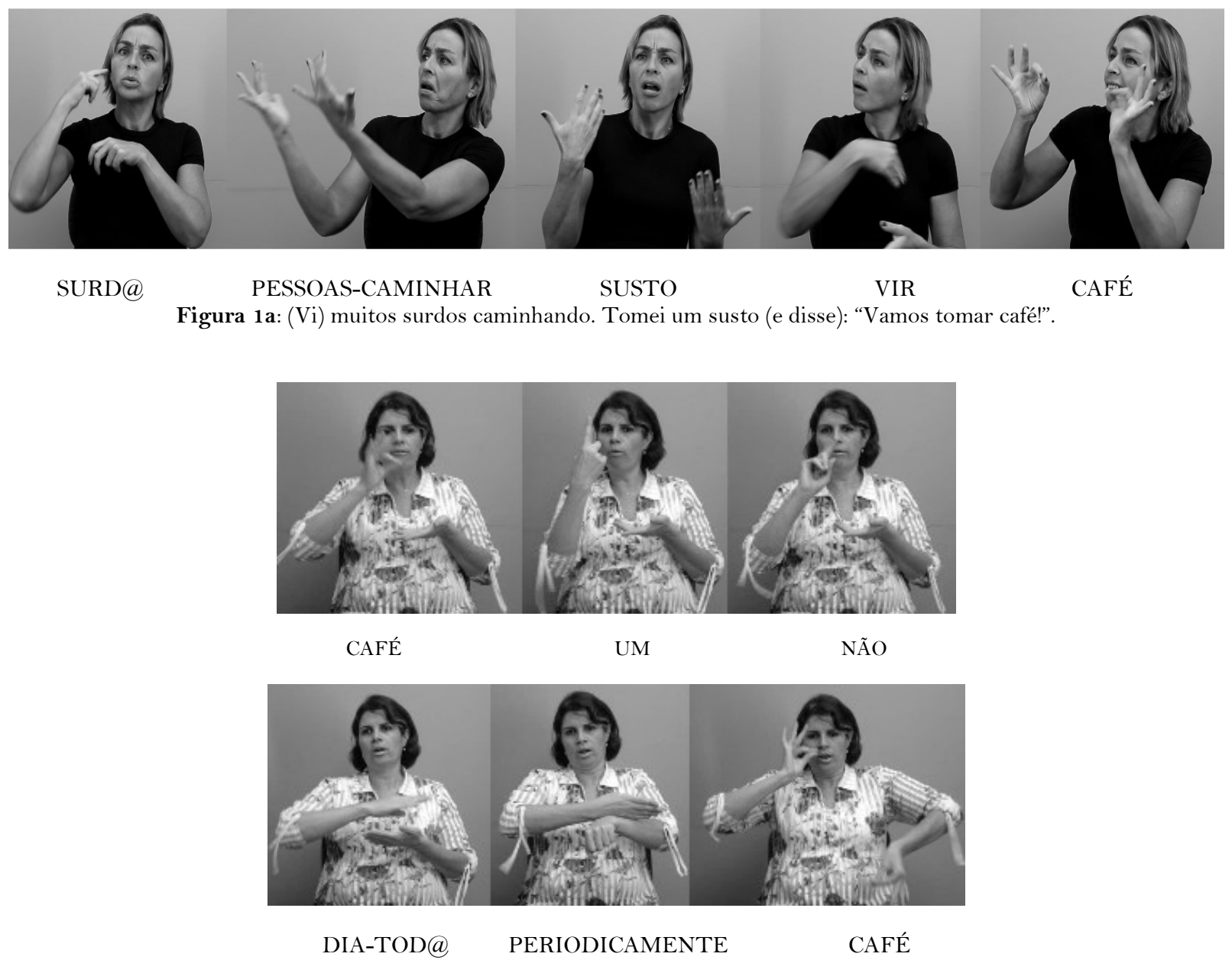

Figura 1b: Não toma só um café. Passa o dia todo tomando café.

Figuras 1a e 1b: CAFÉ

Já o sinal ENTENDER teve seu número de mãos duplicado pela expressão de completude da ação ${ }^{11}$ e pluralidade de seu sujeito. No exemplo de SL, esse sinal foi produzido com duas mãos quando esta simulava perguntar a seu interlocutor se ele finalmente tinha entendido o que ela tinha acabado de contar novamente (Figura 2a). Diferentemente, essa mesma versão foi usada por RA, quando esta simulava uma situação em que perguntava a todos os seus alunos se estes haviam entendido o que ela tinha acabado de explicar (Figura 2b).

\footnotetext{
${ }^{11}$ Esse caso parece se distinguir dos casos de expressão de intensidade, dado que o que está em questão não é o quanto (muito ou pouco) o interlocutor imaginário de SL entendeu, mas se este finalmente entendeu.
} 


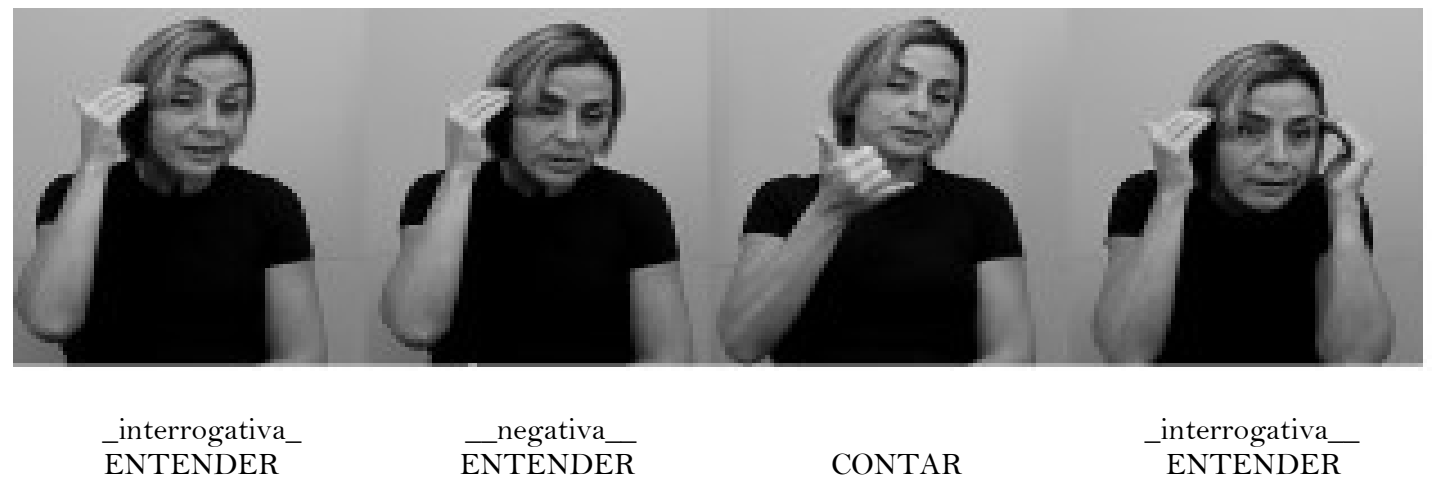

Figura 2a: Perguntei: "Entendeu?” Como ela disse que não, contei (novamente). (Aí) perguntei brava: "Entendeu?”.

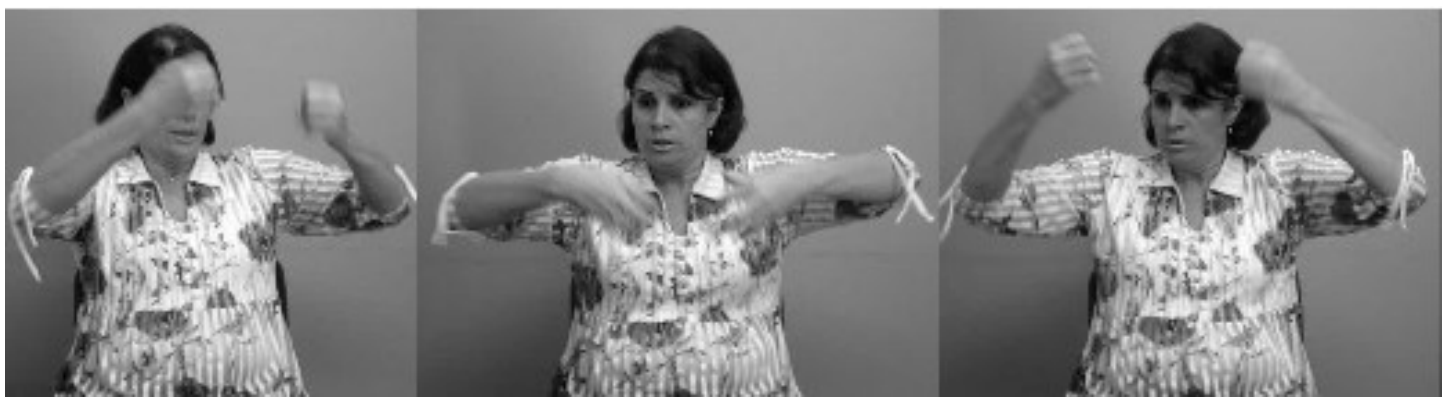

EXPLICAR

EXPLICAR

EXPLICAR

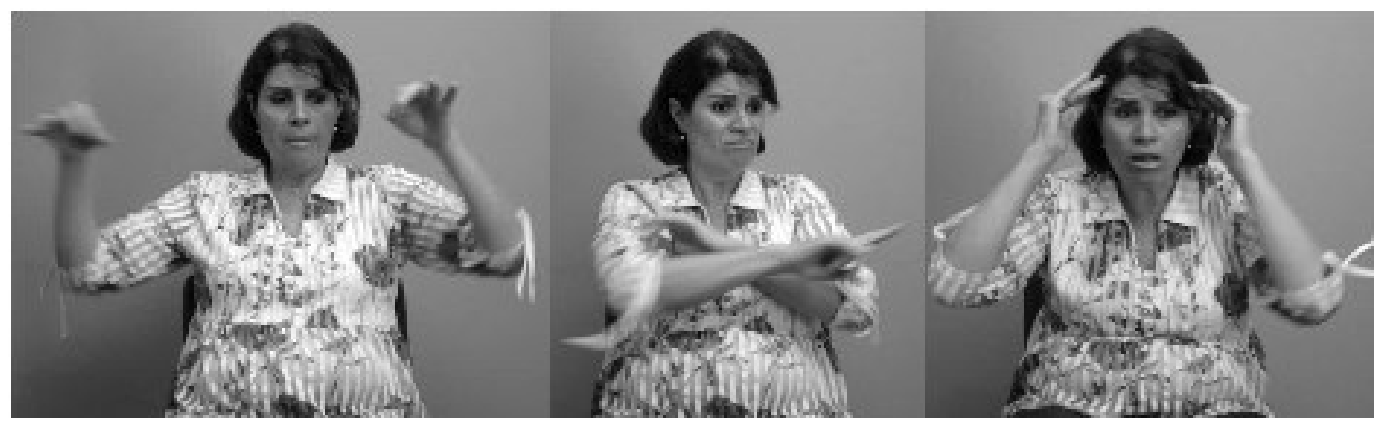

PRONT@_ _interrogativa

Figura 2b: Expliquei para todos (e depois) perguntei: "Vocês entenderam?".

Figuras 2a e 2b: ENTENDER

Por fim, para o sinal VER, os diferentes fatores para a duplicação do seu número de mãos se referem ao tamanho do objeto visto e à pluralidade de seu sujeito. Ambos os casos foram observados nas produções de SL. No exemplo que ilustra a primeira situação, SL, semelhantemente a RA, produz a forma de duas mãos de VER quando se refere à contemplação de um quadro grande, em contraste à contemplação de um quadro pequeno, ao qual ela associa a versão realizada com apenas uma mão (Figura 3a). No entanto, é provável que o uso das duas mãos e de movimento alternado nesse caso indique não apenas a extensão do objeto observado, mas também a ausência de um olhar pontual. Já no exemplo em que a versão com duas mãos de VER expressa pluralidade de seu sujeito, SL, ao contrastar as habilidades visuais de surdos e ouvintes, emprega a versão com duas mãos desse sinal para dizer que o campo visual de ouvintes é limitado, dado que cada um de seus olhos apenas focaliza o que está à sua frente (Figura 3b). É digno de nota que nesse caso as mãos se movem simultaneamente. 

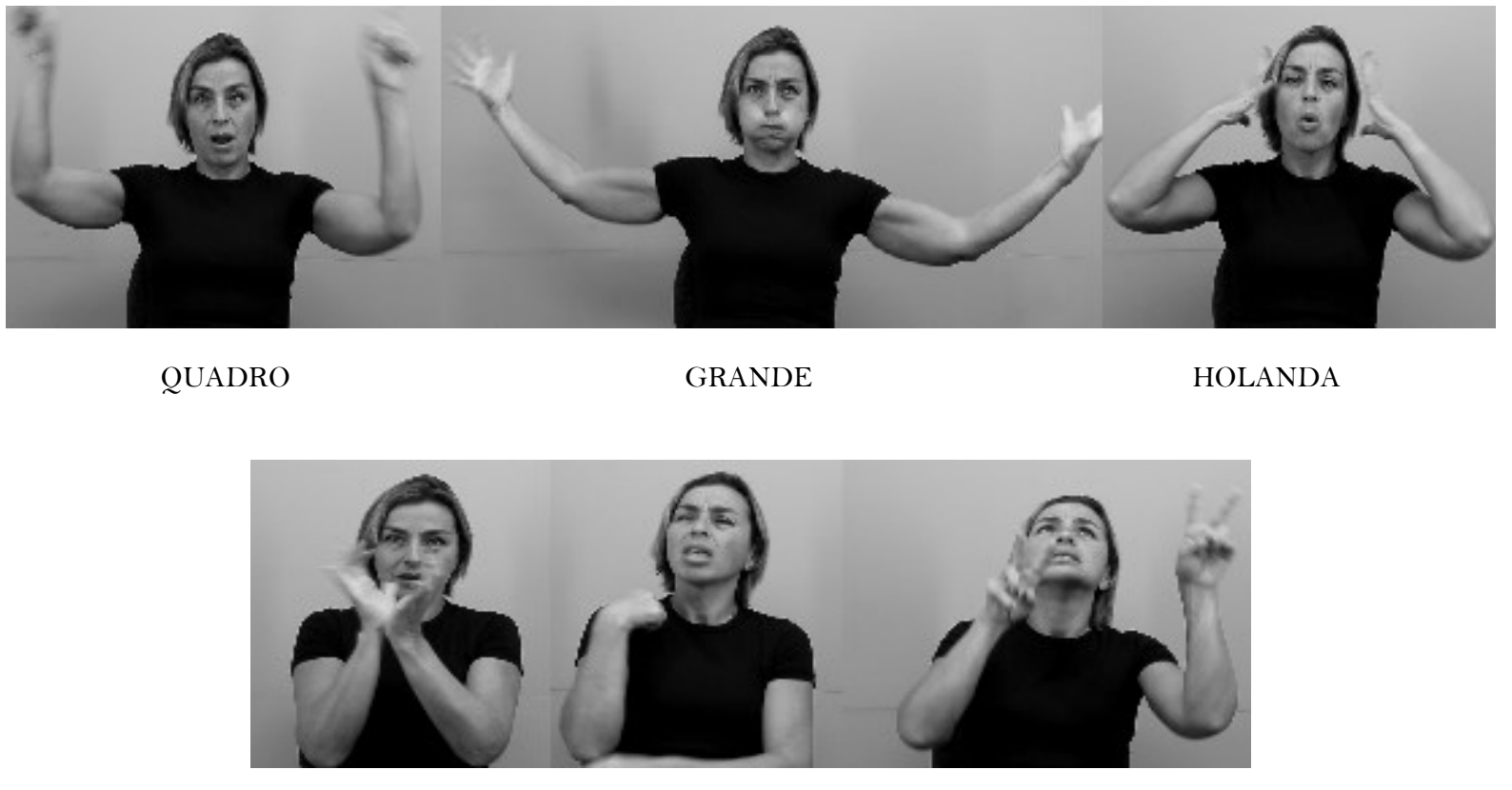

GRUPO

$\mathrm{EU}$

VER

Figura 3a: Eu (fiquei) olhando um grande quadro (pintado por pintores) holandeses.

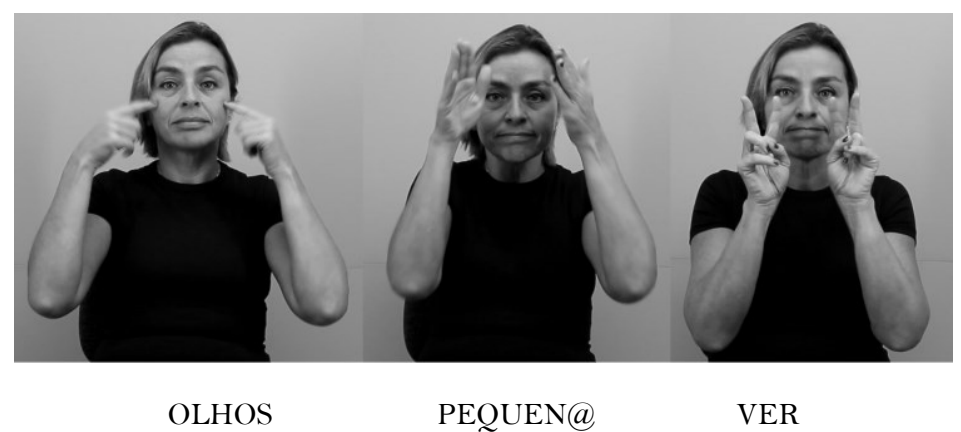

Figura 3b: O olhar é limitado. Os olhos só olham para frente.

Figuras 3a e 3b: VER

Identificamos três outros casos, MAGR@, RIR (versão feita com as duas mãos em contato com o queixo) e DIFERENTE, que, apesar de terem sua duplicação aparentemente motivada por um único fator, não se enquadram nos padrões até então observados.Em MAGR@, semanticamente caracterizável como um estado, a duplicação do número de mãos tem como resultado um sinal que expressa um processo, daí sua possível tradução para o português ser 'emagrecer'. Inversamente, em RIR, semanticamente caracterizável como uma ação, a forma duplicada se refere a um estado, daí sua possível tradução para o português ser 'simpático(a)'. Finalmente, em DIFERENTE, a realização com uma ou duas mãos parece corresponder, respectivamente, à referência explícita ou não a um ou dois referentes ${ }^{12}$.

\section{CONCLUSÃO}

O estudo mostrou que, apesar de na maioria dos casos haver coincidência entre os sujeitos no fator que motivou

\footnotetext{
${ }^{12}$ A forma com uma mão de DIFERENTE normalmente acompanha a oralização da expressão 'não é' do português. Nos exemplos tanto de SL quanto de RA, ela foi empregada quando ambas diziam que determinada coisa não correspondia ao que se dizia ou se perguntava a seu respeito, sendo portanto, diferente disso.
} 
o emprego da forma de duas mãos dos estímulos, houve casos discrepantes. Tal fato corrobora a hipótese levantada por Xavier e Barbosa (2013) e testada aqui, segundo a qual a duplicação do número de mãos de um mesmo sinal pode resultar de diferentes motivações semânticas. O estudo em discussão confirmou a duplicação no número de mãos associada à expressão de pluralidade, de aspecto e intensidade, como previsto por Xavier e Barbosa. Além disso, ele também revelou fatores semânticos não citados pelos autores, a saber: a expressão da extensão do objeto a que o sinal faz referência ou ainda o olhar não pontual a este.

\section{AGRADECIMENTOS}

Agradecemos ao Conselho Nacional de Desenvolvimento Científico (CNPq) por financiar este estudo (Processo 151395/2010-1) e às nossas colaboradoras surdas Regiane Agrella e Sylvia Lia Grespan Neves não apenas pela participação no estudo, mas também por nos permitir usar sua imagem para ilustração de nossos dados. Por fim, agradecemos à FENEIS-SP por ter nos permitido realizar a coleta de dados em seu estúdio.

\section{REFERÊNCIAS}

BATTISON, Robbin. Lexical borrowing in American Sign Language. Silver Spring, MD: Linstok, 1978. Phonological deletion in American Sign Language. Sign Language Studies, v. 5, p. 1-19. 1974.

HENDRIKS, Bernadet. Simultaneous use of two hands in jordanian sign language. In: VERMEERBERGEN, Myriam, et al. (Org.). Simultaneity in signed languages: form and function. Amsterdam: John Benjamins Publishing Company, 2007. p 237-255.

JOHNSTON, Trevor; SCHEMBRI, Adam. Australian sign language (Auslan): an introduction to sign language linguistics. Cambridge: Cambridge University Press, 2007.

KLIMA, Edward; BELLUGI, Ursulla. The signs of language. Cambridge: Harvard University Press, 1979.

LIDDELL, S. K. Grammar, gesture and meaning in American sign language. Cambridge: Cambridge University Press,2003.

LIDDELL, Scott. K.; JOHNSON, Robert E. American sign language: the phonological base. Sign Language Studies, v. 64, p. 197-277, 1989.

NILSSON, Anna-Lena. The non-dominant hand in a Swedish sign language discourse. VERMEERBERGEN, Myriam; et al. (Org.). Simultaneity in signed languages: form and function. Amsterdan: John Benjamins Publishing Company, 2007. p. 163-185.

SANDLER, Wendy. Phonology, phonetics, and the nondominant hand. In: GOLDSTEIN, Louis, et al. (Org.). Papers in laboratory phonology: varieties of phonological competence. Berlin: Mouton-de Gruyter, 2006. p. 185-212.

VERMEERBERGEN, Myriam; DEMEY, Eline. Sign + gesture $=$ speech + gesture? In: VERMEERBERGEN, Myriam; et al. (Org.). Simultaneity in signed languages: form and function. Amsterdam: John Benjamins Publishing Company, 2007. p. 257-282.

XAVIER, André Nogueira. Variação fonológica na libras: um estudo da alternância no número de articuladores manuais envolvidos na produção dos sinais. In: SEMINÁRIOS DE TESES EM ANDAMENTO, 16, Campinas, 2011. Anais ... Campinas: Unicamp, 2011. v. 5. p. 119-145. 
Doubling of the number of hands as a resource for the expression of meaning intensification in Brazilian Sign Language (Libras). Journal of Speech Sciences, São Paulo, v. 1, p. 169-181,2013.

XAVIER, André Nogueira. Descrição fonético-fonológica dos sinais da língua brasileira de sinais (libras). 145fls. Dissertação (Mestrado em Linguística) - Faculdade de Filosofia, Letras e Ciências Humanas, Universidade de São Paulo, 2006.

XAVIER, André Nogueira; BARBOSA, Plínio Almeida. Com quantas mão se faz um sinal? Um estudo do parâmetro número de mãos na produção de sinais da língua brasileira de sinais (libras). Todas as Letras, São Paulo, v. 15, n. 1, p. 111-128, 2013.

Recebido em 29/os/2014. Aprovado em16/o6/15. 